\title{
Intraoperative brief electrical stimulation (BES) for prevention of shoulder dysfunction after oncologic neck dissection: study protocol for a randomized controlled trial
}

Brittany Barber ${ }^{1 *}$, Margaret McNeely ${ }^{2}$, K. Ming Chan ${ }^{3}$, Rhys Beaudry ${ }^{2}$, Jaret Olson ${ }^{4}$, Jeffrey Harris ${ }^{1}$, Hadi Seikaly ${ }^{1}$ and Daniel $\mathrm{O}^{\prime}$ Connell

\begin{abstract}
Background: Shoulder pain and dysfunction are common after oncologic neck dissection for head and neck cancer (HNC), due to traction, compression, and devascularization injuries to the spinal accessory nerve (SAN). Shoulder pain and dysfunction can hinder postoperative rehabilitation and hygiene, activities of daily living (ADLs), and return to work after treatment for HNC. Due to the rising incidence of human papillomavirus (HPV)-associated oropharyngeal cancer, patients are often diagnosed in the third or fourth decade of life, leaving many potential working years lost if shoulder dysfunction occurs. Brief electrical stimulation (BES) is a novel technique that has been shown to enhance and accelerate neuronal regeneration after injury through a brain-derived neurotrophic growth factor (BDNF)-driven molecular pathway in multiple peripheral nerves in both humans and animals.

Methods/Design: This is a randomized controlled trial testing the effect of intraoperative BES on postoperative shoulder pain and dysfunction. All adult participants with a new diagnosis of HNC undergoing surgery with neck dissection, including Level Illb and postoperative radiotherapy, will be enrolled. Participants will undergo intraoperative BES after completion of neck dissection for 60 min continuously at $20 \mathrm{~Hz}, 3$ to $5 \mathrm{~V}$, in 100-msec pulses. Postoperatively, participants will be evaluated using the Constant-Murley Shoulder Score, a scale that assesses shoulder pain, ADLs, strength, and range of motion. Secondary outcomes measured will include nerve conduction studies (NCS) and electromyographic (EMG) studies, as well as scores on the Oxford Shoulder Score (OSS), the Neck Dissection Impairment Index (NDII), and the University of Washington Quality of Life (UW-QOL) score. Primary and secondary outcomes will be assessed at 6 weeks, 3 months, 6 months, and 12 months.

Discussion: The objective of this study is to evaluate the effect of BES on postoperative clinical and objective shoulder functional outcomes and pain after oncologic neck dissection. BES has been shown to be successful in accelerating peripheral nerve regeneration in both animal and human participants in multiple different peripheral nerves. If successful, this technique may provide an adjunctive prevention option for shoulder pain and dysfunction in HNC patients.
\end{abstract}

Trial registration: NCT02268344: 17 October 2014.

Keywords: Neck dissection, Electrical stimulation, Head neck cancer, Nerve regeneration, Axonal regeneration, Spinal accessory nerve

\footnotetext{
* Correspondence: brittanybarber0@gmail.com

${ }^{1} 1 \mathrm{E} 4$, Walter Mackenzie Center, University of Alberta Hospital, 8440-112 St,

Edmonton, AB T6G 2B7, Canada

Full list of author information is available at the end of the article
} 


\section{Background}

Head and neck cancer (HNC) is now the fifth most common cancer in the world due to the rising incidence of oropharyngeal cancer (OPC) from increased transmission of the human papillomavirus (HPV) [1]. HPV-associated oropharyngeal cancer (HPV-OPC) most commonly occurs in patients in the third or fourth decade of life [2]. Advanced $\mathrm{HNC}$, including OPC, is often treated with resection, which includes a neck dissection, or extirpation of lymph nodes known to be, or possibly infiltrated, by locoregional cancer spread [3].

During the neck dissection, Levels II and V are often dissected. The spinal accessory nerve (SAN) courses through these levels, and thus, is also subject to dissection. Often, retraction and manipulation of the SAN, which innervates the trapezius and sternocleidomastoid muscles, is necessary to access Levels II and V [4]. Furthermore, the superior $5 \mathrm{~cm}$ of the SAN is often devascularized in a Level IIb dissection in order to skeletonize all lymphatic tissues off the nerve [5].

Devascularization and retraction of the SAN results in axonal injury, which can give rise to shoulder pain and dysfunction postoperatively, even in nerve-sparing procedures [6]. Particularly, thorough dissection of Level IIb and Level V can contribute to these symptoms [7]. Shoulder pain and dysfunction from SAN injury has pronounced negative effects on quality of life, which are well-documented [8]. Furthermore, as the majority of HNC patients are male and still of working age [9], the ramifications of shoulder pain and dysfunction can also have a socioeconomic impact.

In the last two decades, it has been demonstrated, in both humans and animal models, that application of intraoperative brief electrical stimulation (BES) to transected motor and sensory nerves promotes axonal outgrowth and, thereby, enhances nerve regeneration [10]. In these studies of motoneurons, $60 \mathrm{~min}$ of $\mathrm{BES}$ at $20 \mathrm{~Hz}$ was shown to be as effective as continuous stimulation for 2 weeks, suggesting that BES should be a clinically viable technique [10].

A randomized control trial in human participants with carpal tunnel syndrome (CTS) was initiated at the University of Alberta. BES was applied for $1 \mathrm{~h}$ following carpal tunnel release and compared to a sham control group using motor unit number estimation (MUNE) and sensory and motor nerve conduction studies (NCS). Six months after BES was applied, MUNE was found to be significantly higher in the BES group, and terminal motor latency and sensory nerve conduction values improved significantly faster than in controls [11].

Given the previously demonstrated success of this technique in other motor nerves, we elected to examine its application to the SAN in a patient population who are in the prime of their working years.

\section{Objective}

The aim of this study is to assess the efficacy of BES on postoperative clinical and objective shoulder functional outcomes and pain in HNC patients undergoing oncologic neck dissection.

\section{Hypothesis}

We hypothesize that the use of intraoperative BES will enhance recovery of the SAN and decrease shoulder pain and dysfunction after oncologic neck dissection.

\section{Methods/Design}

The study will be conducted as a double-blinded, randomized controlled trial. Participants will be identified for eligibility from the Northern Alberta Head and Neck Tumor Board (NAHNTB), and will be recruited from the University of Alberta Otolaryngology Clinic after diagnosis of HNC has been confirmed. All surgeries will take place at the University of Alberta Hospital. Informed written consent will be obtained from each participant prior to enrollment in the study and will be sought at the time of surgical booking. Two parallel treatment groups will be examined with a 1:1 allocation: 1) Brief intraoperative electrical stimulation continuously at $20 \mathrm{~Hz}, 3$ to $5 \mathrm{~V}$, at 0.1-msec pulses for $60 \mathrm{~min}$, or 2) No stimulation.

\section{Subjects}

Participants will undergo a screening recruitment checklist to determine eligibility for the study.

\section{Eligibility criteria \\ Inclusion criteria}

- Age $>18$ years

- New diagnosis of head and neck cancer

- Undergoing major resection and reconstruction surgery with oncologic neck dissection including Level IIb or V

\section{Exclusion criteria}

- Oncologic resection necessitating unilateral or bilateral resection of the sternocleidomastoid, SAN, or partial resection of trapezius muscle due to tumor invasion

- Previous surgery or radiation therapy to the head and/or neck

- Recurrent head and neck cancer

- Pre-existing shoulder pain, dysfunction or weakness, including myopathy, neuropathy, or arthropathy at the time of the pre-operative assessment

- Presence of existing implanted electrical device (for example, pacemaker, deep brain stimulator, vagal nerve stimulator) 
- Previous or current neurological disease that may adversely affect shoulder dysfunction

- Unable to read, write, and speak English

- Lacking capacity to give consent

- Unwilling to present for follow-up appointments or perform follow-up objective shoulder assessment

\section{Randomization}

Participants will be randomized to receive: 1) BES, or 2) No Stimulation, with a 1:1 allocation scheme. The randomization sequence was computer-generated, assignments placed on cards and concealed in sequentially numbered sealed opaque envelopes. After general anesthesia has been administered, a card will be removed from the respective opaque envelope by the circulating operating room nurse, who is not involved in the study. This card will dictate the treatment as stated above. If oncologic neck dissection including Level IIb will be performed bilaterally, the neck with the most extensive nodal burden in Level IIb will be selected for randomization. This will be decided based on preoperative imaging and confirmed with intraoperative findings prior to randomization.

\section{Blinding}

Randomization and treatment will occur in the operating room on the day of surgery. All participants will be blinded to the study group into which they have been placed. The physiotherapist and neurophysiologist who will be performing the study assessments including administering the patient-reported questionnaires will also be blinded to the treatment received by the participant, and will not be present in the operating room at the time of randomization or treatment. Therefore, the only study investigators aware of study group allocation for each participant will be the surgeons, who are not involved in measuring outcomes.

\section{Primary outcome measure}

The primary outcome will be a clinical assessment using the Constant-Murley Shoulder Score, a valid and reliable 100-point scale composed of individual parameters assessing shoulder function and pain [12,13]. This assessment tool includes four subscales that assess pain, activities of daily living (ADLs), strength, and range of motion. A higher score indicates higher functioning. A physiotherapist (MM) will assess shoulder function using this scale. Assessments will occur pre-operatively and at 6 weeks, 3, 6 , and 12 months postoperatively.

\section{Secondary outcome measures}

Secondary outcomes will include both self-report outcomes as well as objective measures of SAN function. Self-report measures include the Oxford Shoulder Score (OSS), the Neck Dissection Impairment Index (NDII), and the University of Washington Quality of Life (UWQOL) questionnaire. The OSS is a 12-item, self-report, validated questionnaire regarding shoulder-specific daily activity in the previous 4 weeks [14-16]. The NDII is a validated ten-item self-report questionnaire using a fivepoint Likert scale for assessment of shoulder and neck discomfort as it relates to ADLs [17]. Quality of life will be assessed on the UW-QOL, a validated self-report questionnaire detailing quality of life in 12 domains specific to head and neck cancer symptoms [18-20]. Objective measures of nerve function will include NCS and electromyographic (EMG) studies and will be completed by a neurophysiologist (KMC). All secondary outcomes will be assessed pre-operatively and at 6 weeks, 3, 6, and 12 months postoperatively.

\section{Interventions \\ Group 1: BES group}

Participants randomized to the BES group will undergo intraoperative BES immediately post-neck dissection continuously at $20 \mathrm{~Hz}$, at 3 to $5 \mathrm{~V}$, in $100-\mathrm{msec}$ pulses for $60 \mathrm{~min}$. Electrical stimulation will be performed by encircling the nerve with fine wire electrodes placed at (anode) and just proximal to (cathode) the region of the nerve under maximal traction, and will be executed using the Grass SD9 Stimulator (Quincy, MA, USA). Voltage will be titrated in an ascending fashion from 1 to a maximum of $5 \mathrm{~V}$ in order to obtain a palpated contraction of the trapezius muscle.

\section{Group 2: control (no stimulation) group}

Participants randomized to the control group will receive no stimulation, as per current standard of care.

\section{Sample size justification}

A sample size of 30 participants in each group will be sufficient to detect an $11 \%$ difference in the ConstantMurley Shoulder Score from the 6-week postoperative time point to the 6-month follow-up in favor of the BES group (power of $80 \%$; significance: $5 \%$ ). In the postoperative period, participants can be expected to exhibit a significant decrease in the Constant-Murley Shoulder Score from the pre-operative assessment [8]. For each participant the percentage improvement will be relative to his/her pre-operative score. We are assuming an improvement of $36 \%$ in the BES group, and a $25 \%$ improvement in the control group at the 6-month follow-up. The sample size will be increased to 36 participants per group to account for an attrition rate of $20 \%$ and to allow adequate power for analysis secondary outcomes. The 6-month follow-up was chosen as the primary time end point based on findings from previous research at the University of Alberta demonstrating 
efficacy of intraoperative BES on median nerve recovery following carpal tunnel release at this time point.

\section{Statistical analysis}

Baseline medical and demographic characteristics, surgical type (MRND versus SND) and adverse events of the two groups will be compared using an independent $t$-test for continuous data and Pearson's Chi-square tests for categorical data. The primary analysis will compare the 2 groups with regard to percent change in the ConstantMurley Shoulder Score from the 6-week postoperative time point to the 6-month follow-up using an independent $t$-test. Secondary outcomes and time points will be analyzed by repeated measures modeling and independent $t$-tests on change scores.

\section{Safety and potential risks}

The risk associated with this procedure is a theoretical risk of increased pain in the immediate postoperative period due to the electrical stimulation. During this time, the participant will be administered pain medication as is standard protocol for all patients undergoing major resection and reconstruction surgery, given the extent of the resection and free flap elevation.

The safety parameters that will be monitored in this study are vital signs and arrhythmias, and any adverse event (AE) or serious adverse event (SAE). Specifically, an $\mathrm{AE}$ may be defined as any adverse reactions that can be specifically attributed to the use of electrical stimulation (for example, charring, visible nerve injury). A SAE may be defined as any $\mathrm{AE}$ that can be specifically attributed to the use of electrical stimulation and will include, but is not limited to, cardiac arrhythmias causing instability, or cardiac arrest. No AE or SAE has occurred in any previous $\mathrm{BES}$ trials involving humans or animals.

\section{Trial management}

The Trial Steering Committee (TSC) in accordance with Good Clinical Practice Guidelines will manage the trial (Chair: DO). All lead investigators and authors of this paper will be TSC members. The Trial Management Committee (BB, DO, MC, MM, RB) will be responsible for the day-to-day running of the trial. A Data Safety Monitoring Board (DSMB) has been assembled and approved to monitor AEs and SAEs. The DSMB will be responsible for safeguarding the interests of the trial participants, potential participants, and investigators.

\section{Research team responsibilities}

KMC is a neurophysiologist and Professor in the Division of Physical Medicine and Rehabilitation. He has extensive experience in pioneering and refining the electrical stimulation protocol, and will be responsible for conducting EMG studies and NCS. BB is an otolaryngology-head and neck surgery resident and will be responsible for performing the electrical stimulation process intraoperatively. $\mathrm{MM}$ is a physiotherapist with extensive experience in physical rehabilitation after oncologic neck dissection, and will be performing all clinical shoulder assessments utilizing the Constant-Murley Shoulder Score. RB is a graduate student in rehabilitation medicine who will assist in the data collection of objective and patient-reported outcomes. DO is a microvascular head and neck surgeon and will be responsible for recruiting participants and ensuring self-report questionnaires are completed by participants. HS is a microvascular surgeon and has been instrumental in refining the intraoperative BES technique. $\mathrm{JH}$ and $\mathrm{JO}$ assisted with study design and critique and will be involved in results analysis and manuscript preparation.

\section{Ethics}

This study will be conducted in accordance with Good Practice Guidelines and has received ethical and operational approval (Pro00046671) by the Human Research Ethics Board (HREB) and Northern Alberta Clinical Trials and Research Center (NACTRC) at the University of Alberta.

\section{Discussion}

The primary objective of this study is to evaluate the effect of intraoperative BES on SAN recovery following traction, compression, and devascularization injury during oncologic neck dissection. The SAN is the principal motor innervation to the trapezius muscle, a key stabilizing muscle of the scapula [21]. The trapezius allows use of the arms for lifting, carrying and overhead work. Paralysis or weakness of the trapezius alters the alignment of the neck and shoulder region, and disrupts the normal movement of the shoulder complex [4, 22]. Chronic neck and shoulder dysfunction are common after neck dissection procedures, negatively impacting both quality of life and return to work [23, 24]. Thus, strategies are needed that help reduce the impact of neck dissection procedures on shoulder function while still ensuring optimal oncologic outcomes.

A significant body of evidence has been put forth regarding the success of BES in promoting regeneration following peripheral nerve injuries. To our knowledge, intraoperative BES has not previously been applied to the SAN for the purposes of alleviating pain or preventing shoulder dysfunction, specifically after oncologic neck dissection. However, the use of surface BES in treatment of neuropathic pain, particularly in peripheral nerve disorders, has been well-established [25]. More specifically, a study by Wilson et al. [12] demonstrated a significantly greater reduction in pain with application of percutaneous 
electrical stimulation to the trapezius, supraspinatus, middle deltoid, and posterior deltoid muscles.

The application of BES to a peripheral nerve following injury was initially performed by Nix and Hopf [26], in the soleus nerve of a rabbit after a crush injury. They subsequently reported accelerated recovery of twitch force, tetanic tension, and muscle action potential in the soleus muscle. Thereafter, application of BES to the sciatic nerve proximal to a crush injury demonstrated significantly improved recovery of the toe-spread reflex [27]. Despite evidence of improved and accelerated motor function in previously-injured peripheral nerves, at that time, the mechanism of BES was not yet elucidated.

Further studies demonstrated that, following application of BES to the rat femoral nerve after transection injury and primary repair, significantly increased numbers of motoneurons regenerated into the nerve branches of the rat femoral nerve when compared to a non-stimulated sham control group. This acceleration of function recovery was found to be due to accelerated sprouting of axons across the nerve repair site and not due to an accelerated rate of regeneration [28]. Subsequent studies have suggested that brain-derived neurotrophic factor (BDNF), a key molecule in activating cyclic adenomonophosphate (cAMP) and protein kinase A (PKA), which lead to downstream protein transcription necessary for neurite outgrowth, may be mediating the effect of BES in accelerating motoneuron regeneration [29]. Thus, a rationale for the use of BES clinically is supported by the findings of this research.

\section{Study limitations}

A limitation of our study is the relatively small sample size given the potential heterogeneity in surgical procedures among patients. The sample size may ultimately limit our ability to examine subgroup differences such as the effect on those undergoing modified radical neck dissection compared to selective neck dissection. Should BES prove safe and show preliminary efficacy, exploratory analyses will be carried out on sub-groups undergoing SND and MRND to determine point estimates and measures of variability for the purposes of a larger Phase III study.

The study was powered to detect a minimal clinically significant difference (MCID) in the Constant-Murley Shoulder Score of 10.4 \% (standard deviation: 15) based on previous research following post-surgical rotator cuff injuries [30, 31]. The MCID for the Constant-Murley Shoulder Score following neck dissection procedures is currently not known. A previous study reported average scores in the range of $70 \%$ following neck dissection surgery, with larger deficits seen in those who had undergone more extensive procedures [8]; thus, we hypothesize that an improvement of $10 \%$ would likely be clinically relevant following oncologic neck dissection for head and neck cancer.

A potential confounder in the study is related to pain sustained from operative incisions. However, this is unlikely to confound the outcomes, as we anticipate similar levels of pain in both groups in the early stages. Additionally, the first follow-up measurement (6 weeks postoperatively) falls outside the acute pain stage.

\section{Relevance}

The potential impact of this study is multi-dimensional. First, if successful, this study will confirm findings from previous human trials at the University of Alberta that have demonstrated accelerated axonal regeneration histologically, as well as, clinically improved functioning of both motor and sensory nerves. In addition, evaluation of this technique as a therapy for axonal devascularization injuries will further define its role in diverse peripheral nerve injuries. Furthermore, and most importantly, should this technique be successful, it will provide an additional therapeutic option for the prevention of shoulder pain and dysfunction in HNC patients undergoing oncologic neck dissection.

\section{Trial status}

Ongoing.

\begin{abstract}
Abbreviations
ADLs: activities of daily living; AE: adverse event; BDNF: brain-derived neurotrophic factor; BES: brief electrical stimulation; CAMP: cyclic adenomonophosphate; CTS: carpal tunnel syndrome; DSMB: Data Safety Monitoring Board; EMG: electromyographic studies; HNC: head and neck cancer; HPV: human papillomavirus; HPV-OPC: HPV-associated oropharyngeal cancer; HREB: Human Research Ethics Board; MCID: minimal clinically significant difference; MUNE: motor unit number estimation;

NACTRC: Northern Alberta Clinical Trials Research Center; NAHNTB: Northern Alberta Head and Neck Tumor Board; NCS: nerve conduction studies; NDII: Neck Dissection Impairment Index; OPC: oropharyngeal cancer; OSS: Oxford Shoulder Score; PKA: protein kinase A; SAE: serious adverse event; SAN: spinal accessory nerve; TSC: Trial Steering Committee; UW-QOL: University of Washington Quality of Life questionnaire.
\end{abstract}

\section{Competing interests}

The authors declare that they have no competing interests.

\section{Authors' contributions}

KMC assisted with study design, outcomes measurement, and manuscript revision. DAO assisted with study design, ethical approval, intraoperative procedures, manuscript construction, and manuscript revision. MM assisted with study design, outcomes measurement, and manuscript revision. BB was responsible for funding acquisition, study design, data collection, intraoperative procedures, manuscript construction, and manuscript revision. HS assisted with study design, intraoperative procedures and troubleshooting, data collection, and manuscript revision. JH was responsible for intraoperative procedures and manuscript revision. JO assisted with study design and funding acquisition. All authors read and approved the final manuscript.

\section{Acknowledgements}

Dr. Francois Roy, PhD, for his knowledge contribution regarding study design and manuscript revision. Funding for this study was provided by the University Hospital Foundation Medical Research Competition from the 
University Hospital Foundation at the University of Alberta. No other sources of funding have been provided to the authors.

\section{Author details}

${ }^{1} 1$ E4, Walter Mackenzie Center, University of Alberta Hospital, 8440-112 St, Edmonton, AB T6G 2B7, Canada. ${ }^{2} 3-41$ Corbett Hall, University of Alberta, Edmonton, AB T6G 2G4, Canada. ${ }^{3}$ Center for Neuroscience, 5-005 Katz Group Center, University of Alberta, Edmonton, AB T6G 2E1, Canada. ${ }^{4} 2 \mathrm{D} 3$ Walter Mackenzie Center, University of Alberta, 8440-112 St, Edmonton, AB T6G 2B7, Canada.

Received: 31 August 2014 Accepted: 6 May 2015

Published online: 30 May 2015

\section{References}

1. Ferreira MB, De Souza JA, Cohen EE. Role of molecular markers in the management of head and neck cancers. Curr Opin Oncol. 2011;23(3):259-64. doi:10.1097/Cco.0b013e328344f53a.

2. Biron VL, Mohamed A, Hendzel MJ, Alan Underhill D, Seikaly H. Epigenetic differences between human papillomavirus-positive and -negative oropharyngeal squamous cell carcinomas. J Otolaryngol Head Neck Surg. 2012;41(1):S65-70.

3. Pfister DG, Ang KK, Brizel DM, Burtness BA, Busse PM, Caudell JJ, et al. Head and neck cancers, version 2.2013. Featured updates to the NCCN guidelines. J Natl Compr Canc Netw. 2013;11(8):917-23.

4. Umeda M, Shigeta T, Takahashi H, Oguni A, Kataoka T, Minamikawa T, et al. Shoulder mobility after spinal accessory nerve-sparing modified radical neck dissection in oral cancer patients. Oral Surg Oral Med Oral Pathol Oral Radiol Endod. 2010;109(6):820-4. doi:10.1016/j.tripleo.2009.11.027.

5. Celik B, Coskun H, Kumas FF, Irdesel J, Zarifoglu M, Erisen L, et al. Accessory nerve function after level $2 \mathrm{~b}$-preserving selective neck dissection. Head Neck. 2009;31(11):1496-501. doi:10.1002/hed.21112.

6. Van Wilgen CP, Dijkstra PU, van der Laan BF, Plukker JT, Roodenburg JL. Shoulder complaints after nerve sparing neck dissections. Int J Oral Maxillofac Surg. 2004;33(3):253-7. doi:10.1006/ijom.2003.0507.

7. Parikh S, Tedman BM, Scott B, Lowe D, Rogers SN. A double blind randomised trial of Ilb or not llb neck dissections on electromyography, clinical examination, and questionnaire-based outcomes: a feasibility study. Br J Oral Maxillofac Surg. 2012;50(5):394-403. doi:10.1016/j.bjoms.2011.09.007.

8. Chepeha DB, Taylor RJ, Chepeha JC, Teknos TN, Bradford CR, Sharma PK, et al. Functional assessment using Constant's shoulder scale after modified radical and selective neck dissection. Head Neck. 2002;24(5):432-6. doi:10.1002/hed.10067.

9. Cancer in New South Wales: incidence, mortality and prevalence report 2005. Cancer Institute NSW; 2005.

10. Gordon T, Brushart TM, Amirjani N, Chan KM. The potential of electrical stimulation to promote functional recovery after peripheral nerve injury-comparisons between rats and humans. Acta Neurochir Suppl. 2007;100:3-11.

11. Gordon T, Amirjani N, Edwards D, Chan KM. Brief post-surgical electrical stimulation accelerates axon regeneration and muscle reinnervation without affecting the functional measures in carpal tunnel syndrome patients. Exp Neurol. 2010;223(1):192-202. doi:10.1016/j.expneurol.2009.09.020.

12. Wilson R, Gunzler D, Bennett M, Chae J. Peripheral nerve stimulation compared with usual care for pain relief of hemiplegic shoulder pain: a randomized controlled trial. Am J P M R Assoc Acad Psychiatrists. 2014;93(1):17-28. doi:10.1097/phm.0000000000000011.

13. Rocourt MH, Radlinger L, Kalberer F, Sanavi S, Schmid NS, Leunig M, et al. Evaluation of intratester and intertester reliability of the constant-murley shoulder assessment. J Shoulder Elbow Surg. 2008;17(2):364-9. doi:10.1016/j.jse.2007.06.024.

14. Angst F, Schwyzer HK, Aeschlimann A, Simmen BR, Goldhahn J. Measures of adult shoulder function: Disabilities of the Arm, Shoulder, and Hand Questionnaire (DASH) and its short version (QuickDASH), Shoulder Pain and Disability Index (SPADI), American Shoulder and Elbow Surgeons (ASES) Society Standardized Shoulder Assessment Form, Constant (Murley) Score (CS), Simple Shoulder Test (SST), Oxford Shoulder Score (OSS), Shoulder Disability Questionnaire (SDQ), and Western Ontario Shoulder Instability Index (WOSI). Arthritis Care Res. 2011;63 Suppl 11:S174-88. doi:10.1002/acr.20630.
15. Dawson J, Rogers K, Fitzpatrick R, Carr A. The Oxford shoulder score revisited. Arch Orthop Trauma Surg. 2009;129(1):119-23. doi:10.1007/s00402-007-0549-7.

16. Younis F, Sultan J, Dix S, Hughes PJ. The range of the Oxford shoulder score in the asymptomatic population: a marker for post-operative improvement. Ann R Coll Surg Engl. 2011;93(8):629-33. doi:10.1308/003588411X13165261994193.

17. Taylor RJ, Chepeha JC, Teknos TN, Bradford CR, Sharma PK, Terrell JE, et al. Development and validation of the neck dissection impairment index: a quality of life measure. Arch Otolaryngol Head Neck Surg. 2002;128(1):44-9.

18. D'Cruz AK, Yueh B, Das AK, McDowell JA, Chaukar DA, Ernest AW. Validation of the University of Washington quality of life questionnaires for head and neck cancer patients in India. Indian J Cancer. 2007;44(4):147-54.

19. De Andrade FP, Biazevic MG, Toporcov TN, Togni J, De Carvalho MB, Antunes JL. Discriminant validity of the University of Washington quality of life questionnaire in the Brazilian context. Rev Bras Epidemiol. 2012;15(4):781-9.

20. Rogers SN, Scott B, Lowe D. An evaluation of the shoulder domain of the University of Washington quality of life scale. Br J Oral Maxillofac Surg. 2007;45(1):5-10. doi:10.1016/j.bjoms.2006.09.007.

21. McNeely ML, Parliament M, Courneya KS, Seikaly H, Jha N, Scrimger R, et al. A pilot study of a randomized controlled trial to evaluate the effects of progressive resistance exercise training on shoulder dysfunction caused by spinal accessory neurapraxia/neurectomy in head and neck cancer survivors. Head Neck. 2004;26(6):518-30. doi:10.1002/hed.20010.

22. Brown $H$, Burns $S$, Kaiser CW. The spinal accessory nerve plexus, the trapezius muscle, and shoulder stabilization after radical neck cancer surgery. Ann Surg. 1988;208(5):654-61.

23. Donatelli-Lassig AA, Duffy SA, Fowler KE, Ronis DL, Chepeha DB, Terrell JE. The effect of neck dissection on quality of life after chemoradiation. Otolaryngol Head Neck Surg. 2008;139(4):511-8. doi:10.1016/j.otohns.2008.07.007.

24. Terrell JE, Welsh DE, Bradford CR, Chepeha DB, Esclamado RM, Hogikyan ND, et al. Pain, quality of life, and spinal accessory nerve status after neck dissection. Laryngoscope. 2000;110(4):620-6. doi:10.1097/00005537-200004000-00016.

25. Slavin KV. Peripheral nerve stimulation for neuropathic pain. Neurotherapeutics: J Am Soc Exp NeuroTherapeutics. 2008;5(1):100-6. doi:10.1016/j.nurt.2007.11.005.

26. Levy O, Haddo O, Massoud S, Mullett H, Atoun E. A patient-derived constant-murley score is comparable to a clinician-derived score. Clin Orthop Relat Res. 2014;472(1):294-303. doi:10.1007/s11999-013-3249-3.

27. Nix W, Hopf H. Electrical stimulation of regenerating nerve and its effect on motor recovery. Brain Res. 1983;272(1):21-5.

28. Pockett S, Gavin RM. Acceleration of peripheral nerve regeneration after crush injury in rat. Neurosci Lett. 1985;59(2):221-4.

29. Al-Majed AA, Neumann CM, Brushart TM, Gordon T. Brief electrical stimulation promotes the speed and accuracy of motor axonal regeneration. J Neurosci. 2000;20(7):2602-8.

30. Al-Majed AA, Brushart TM, Gordon T. Electrical stimulation accelerates and increases expression of BDNF and trkB mRNA in regenerating rat femoral motoneurons. Eur J Neurosci. 2000;12(12):4381-90.

31. Grassi FA, Tajana MS. The normalization of data in the constant-murley score for the shoulder. A study conducted on 563 healthy subjects. Chir Organi Mov. 2003;88(1):65-73.

\section{Submit your next manuscript to BioMed Central and take full advantage of:}

- Convenient online submission

- Thorough peer review

- No space constraints or color figure charges

- Immediate publication on acceptance

- Inclusion in PubMed, CAS, Scopus and Google Scholar

- Research which is freely available for redistribution 